\title{
Effect of tooth-brushing with a microcurrent on dentinal tubule occlusion
}

\author{
Gangjae KIM¹, Byoung-Duck ROH², Sung-Ho PARK², Su-Jung SHIN³ and Yooseok SHIN² \\ ${ }^{1}$ Department of Conservative Dentistry, College of Dentistry, Yonsei University, 50-1 Yonsei-ro, Seodaemun-gu, Seoul 120-752, Korea \\ ${ }^{2}$ Department of Conservative Dentistry and Oral Science Research Center, College of Dentistry, Yonsei University, 50-1 Yonsei-ro, Seodaemun-gu, \\ Seoul 120-752, Korea \\ ${ }^{3}$ Department of Conservative Dentistry and Oral Science Research Center, College of Dentistry, Gangnam Severance Hospital, Yonsei University, \\ 211 Eonju-ro, Gangnam-gu, Seoul 135-720, Korea \\ Corresponding author, Yooseok SHIN; E-mail: densys@yuhs.ac
}

The purpose of this study was to investigate whether tooth-brushing with a microcurrent was effective in inducing dentinal tubule occlusion. The specific aims of the study were (1) to evaluate the effectiveness of tooth-brushing with a microcurrent on dentinal tubule occlusion by using scanning electron microscopy (SEM); and (2) to compare the dentinal fluid flow rate after tooth-brushing with a microcurrent by using a sub-nanoliter-scaled fluid flow measuring device (NFMD). All experimental groups showed partially occluded dentinal tubules and crystal-like structures at a specific microcurrent intensity indicated that tooth-brushing with a microcurrent could efficiently occlude dentinal tubules. The decrease in dentinal fluid flow rate in the tooth-brushing with microcurrents group indicated that dentinal tubules were occluded and the flow of dentinal fluid had decreased.

Keywords: Dentin hypersensitivity, Dentinal tubule, Microcurrent, Nanoliter-scaled fluid flow measuring device, SEM

\section{INTRODUCTION}

Dentin hypersensitivity is a common disease caused by exposure of the dentinal tubules that allow the movement of intradentinal fluid ${ }^{1)}$. The most commonly accepted hypothesis for explaining pulpal stimulus transmission was provided by the Brannstrom hydrodynamic theory in 1966. Brannstrom proposed that the inflicting stimulus causes dynamic intradentinal fluid flow in dentinal tubules, resulting in deformation of odontoblastic processes along with adjacent nerve fibers and leading to dentin hypersensitivity ${ }^{2}$.

To date, many desensitizing agents have been developed to reduce dentin hypersensitivity, and the most accessible such agent is toothpaste. If daily brushing with a desensitizing toothpaste occludes open dentinal tubules, it could be an effective method to reduce dentin hypersensitivity ${ }^{3)}$. Using scanning electron microscopy (SEM), Ling et al. showed that different desensitizing toothpastes were able to occlude dentinal tubules. They also confirmed that brushing dentin surfaces with toothpastes using many desensitizing agents created smear layers or smear plugs in dentinal tubules that could reduce dentin hypersensitivity ${ }^{4}$.

Although many studies have revealed an abundance of desensitizing agents, no single agent can be considered ideal for long-term relief from dentin hypersensitivity. Therefore, new ways to decrease dentin hypersensitivity were introduced in dentistry, with iontophoresis being one of them. Iontophoresis is a well-known medical treatment technique that increases the skin penetration of medicinal drugs with the same charge by using

Color figures can be viewed in the online issue, which is available at J-STAGE.

Received Jul 9, 2019: Accepted Oct 1, 2019

doi:10.4012/dmj.2019-205 JOI JST.JSTAGE/dmj/2019-205 electrostatic repulsion and allows the simultaneous penetration of neutral molecular chemicals when water is infiltrated using electro-osmosis ${ }^{5}$. Iontophoresis was first used in the early 1960s to treat dentin hypersensitivity ${ }^{6}$. A previous study reported that using iontophoresis treatment with fluoride, dentin hypersensitivity can be reduced by blocking the dentinal tubules, which are coated with $\mathrm{CaF}_{2}{ }^{7}$. Another study suggested that fluoride iontophoresis treatment is effective and safe and significantly decreases dentin hypersensitivity ${ }^{8}$. However, iontophoresis treatment is expensive and requires special equipment, preventing its daily use by patients suffering from dentin hypersensitivity.

Recently, a smart toothbrush using a microcurrent (Microworld, Seoul, Korea) has been developed, and it can be used more easily with similar effects as iontophoresis. The toothbrush contains a chip behind the head. This chip is an ultra-thin membrane-type microcurrent cell and is composed of staneless steel and aluminum. These two metals cause oxidation-reduction reactions with saliva and the fluoride-containing toothpaste. The microcurrent cell and the toothbrushes are electrically connected to supply microcurrents directly to the teeth with an electrolyte. Thus, without an additional source of electric power, when the smart brush is applied with saliva in the mouth, it can generate microcurrents, which are expected to occlude dentinal tubule efficiently. However, no previous studies have assessed the effectiveness of such smart toothbrushes; therefore, in this study we attempted to determine whether dentinal tubules are actually occluded well when brushing with a smart toothbrush and Colgate Total toothpaste (ColgatePalmolive, New York, NY, USA).

Evaluations of the effects of desensitizing agents on 
dentinal tubule occlusion are commonly performed by assessing the occlusion of the dentinal tubules using SEM or by observation of changes in dentin permeability by measuring the hydraulic conductance of a dentin $\operatorname{disc}^{9}$. In this study, dentinal tubule occlusion was observed by using SEM and dentin permeability was measured by a sub-nanoliter-scaled fluid flow measuring device (NFMD).

Assessments of surface topography are commonly performed in dental material science ${ }^{10)}$. Surface roughness measurements are often used to identify changes in tooth structure following erosive wear and to investigate the efficacy of anti-erosion and remineralizing products ${ }^{11}$. Dentinal tubule occlusion can be expected to affect the surface roughness of dentin due to the surface nature of dentin hypersensitivity ${ }^{12}$. Therefore, surface roughness can be a useful indicator of tubule patency.

The purpose of this study was to investigate whether tooth-brushing with a microcurrent was effective in inducing dentinal tubule occlusion and surface roughness. Therefore, the specific aims of the study were (1) to evaluate the effectiveness of tooth-brushing with a microcurrent in inducing dentinal tubule occlusion by using SEM; 2) to compare the surface roughness after tooth-brushing with a microcurrent, and 3) to compare the DFF rates after tooth-brushing with a microcurrent by using an NFMD.

\section{MATERIALS AND METHODS}

\section{Dentin sample preparation}

Thirty extracted human third molars were collected after obtaining informed consent under a protocol approved by the dental hospital (IRB approval no:2-2019-0001). Teeth with caries, cracks, or gross irregularities of dentin structure were excluded from the study. The teeth were cleaned thoroughly and stored in distilled water no longer than a month prior to their use.

Thirty dentin discs were cut perpendicular to the long axis of the tooth each with a thickness of $3.0 \mathrm{~mm}$ above the cemento-enamel junction (CEJ) and $3.0 \mathrm{~mm}$ below the CEJ by using a low-speed diamond cutter (MetsawLS, R\&B, Daejeon, Korea) with water as coolant.

\section{Experimet part 1}

1. Experimental design

After preparation of the specimens, the occlusal surface of each specimen was sanded with 600-grit silicon carbide paper for $30 \mathrm{~s}$ to create a standard smear layer. The smear layer was subsequently removed by etching with $37 \%$ phosphoric acid for $20 \mathrm{~s}$ and rinsing thoroughly with water for $10 \mathrm{~s}$. The etched specimens were kept wet in distilled water to maintain $100 \%$ permeability. Then, the specimens were grouped as follows and treated using Colgate Total.

Group 1: Phosphoric acid-etched specimens without any treatment

Group 2: Phosphoric acid-etched specimens brushed without a microcurrent

Group 3: Phosphoric acid-etched specimens brushed with a microcurrent $(20 \mu \mathrm{A})$

Group 4: Phosphoric acid-etched specimens brushed with a microcurrent $(100 \mu \mathrm{A})$

Group 5: Phosphoric acid-etched specimens brushed with a microcurrent $(200 \mu \mathrm{A})$

Group 6: Phosphoric acid-etched specimens brushed with a microcurrent $(500 \mu \mathrm{A})$

In group 2, a toothbrush (Oral-B, Seoul, Korea) without microcurrents was applied to the dentin surface at an inclination of about $90^{\circ}$ under constant loading at the rate of $40,000 \mathrm{strokes} / \mathrm{min}$ for $1 \mathrm{~min} /$ day in tap water for 7 days. In groups 3-6, the same protocol was applied to the specimens with a smart toothbrush utilizing a microcurrent. After each brushing session, the specimens were washed with distilled water using an ultrasonic bath and kept wet in distilled water.

\section{Observation of dentin surface occlusion by SEM}

One dentin disc for each treatment group was prepared for transverse observation. Specimens were dried in a desiccator and sputter-coated with gold in a vacuum evaporator (EIKO IB-3 ion coater, Hitachi, Tokyo, Japan). Micrographs of the dentin surface were obtained using a scanning electron microscope (JEOL-7800F, JEOL, Tokyo, Japan) on September 3, 2018.

\section{Measurement of surface roughness}

One dentin disc from each group was imaged and analyzed for surface roughness by using the surface profiler (DektakXT Stylus Profiler, Bruker, Bilerica, MA, USA). The surface profiler records the irregularities in the dentin surface as it moves to the end of the specimen, and the $R a$ value is calculated. The $R a$ value is used to detect general variations in overall profile height. A change in $R a$ typically signifies a new variation in the process. Each dentin specimen's $R a$ value was measured and analyzed.

\section{Selection of proper microcurrent}

Preliminary experiments were performed with one specimen per group, which was assessed using SEM and surface profiler. We selected the group that showed maximum dentinal tubule occlusion. Then, we compared the DFF rate in that group with those in groups 1 and 2 by using eight specimens per group.

\section{Experiment part 2}

1. Measurement of the DFF rate by NFMD

The NFMD fabricated for this study consisted of three parts: a glass capillary and photo-sensor to detect fluid movement; a servo motor, lead screw, and ball nut to track fluid movement; and a rotary encoder and computer software to record the data (Fig. 1).

A water-filled glass capillary with an internal diameter of $0.5 \mathrm{~mm}$ was connected between a water reservoir and the tooth. A photo-sensor detected the movement of an air bubble trapped within the capillary, and the minimum measurable volume of water movement was $0.196 \mathrm{~nL}[(0.25 \times 0.25 \mathrm{~mm} \times \pi) \times 1 \mu \mathrm{m}$, while the diameter of the capillary was $0.5 \mathrm{~mm}^{13,14)}$. 


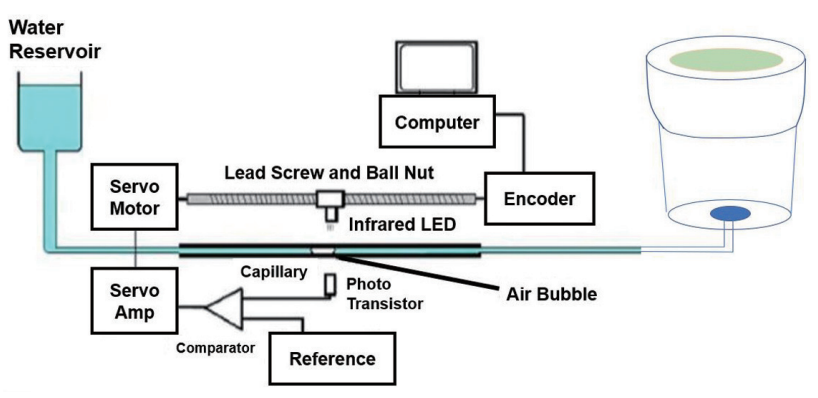

Fig. 1 A Schematic diagram of the fluid flow measurement system (Nanoflow, IB systems, Seoul, Korea) modified from the original publication.

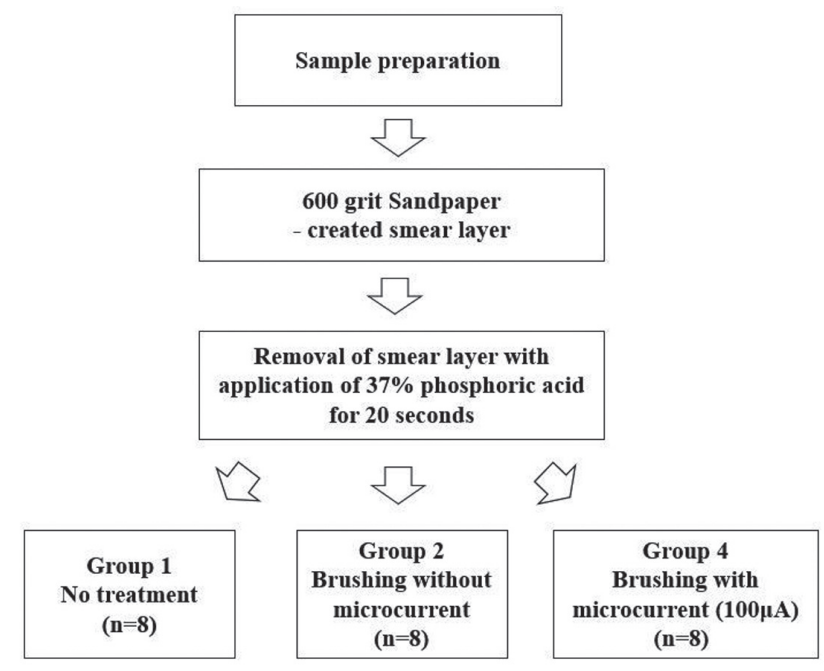

Fig. 2 Summary of the experimental design for preparation of dentin specimens for different treatments to occlude dentinal fluid flow.

After SEM analysis, group 4 was chosen for DFF measurement. Because group 4 showed maximum dentinal tubule occlusion compared to groups 3, 5, 6 . The prepared specimen's pulp tissue was carefully removed without damaging the pre-dentin surface by using an explorer. A metal tube $0.9 \mathrm{~mm}$ in diameter was inserted into the tooth and attached using an adhesive (All-bond Universal, Bisco, Schaumburg, IL, USA) and a flowable composite (Metafil flo, Sun Medical, Shiga, Japan) to confirm that one end of the metal tube was located in the pulp chamber. The outer surface of the bonded interface between the composite resin and the tooth with the metal tube was covered by nail varnish. Finally, the previously prepared specimen was connected to the glass capillary by silicon tubing filled with distilled water (Fig. 1). A hydrostatic pressure of $20 \mathrm{~cm} \mathrm{H}_{2} \mathrm{O}$ was applied throughout all procedures with a water reservoir to simulate physiological pulp pressure. Each specimen had a stabilizing time of $3 \mathrm{~min}$ after being connected to the NFMD, and flow was measured for $5 \mathrm{~min}$. The DFF rate was calculated by dividing the total DFF by 5 $\min ^{13,14)}$. Figure 2 shows summary of the experimental design. And we also compared groups 1, 2, and 4 with a control group that showed occlusion of all outer dentin surfaces. Theoretically, there should be no dentinal fluid flow at all if all of the dentinal tubules are blocked by a substance. So we made a control group that surface of the specimen was blocked by flowable composite.

\section{Statistical analysis}

Statistical analyses were performed with computer software (IBM SPSS Statistics v23.0, IBM, Chicago, IL, USA). One-way ANOVA and Tukey's post-hoc test were used to determine inter-group significance. All statistical analyses were performed at the $95 \%$ confidence level $(p=0.05)$.

\section{RESULTS}

\section{Experimet part 1}

1. Observation of dentin surface occlusion by SEM

1) Phosphoric acid treatment

After application of $37 \%$ phosphoric acid for $20 \mathrm{~s}$, the dentin surfaces were free of smear layer and smear plugs and almost all of the dentinal tubules were open (Figs. 3A and B).

2) Brushing treatment

All tested treatments produced morphological modifications to the dentin surface. All experimental groups used a Colgate Total toothpaste, which contains $0.24 \%$ sodium fluoride, when tooth-brushing the dentin specimen. Dentifrice abrasives can have an occluding effect on dentinal tubules. This phenomenon occurs via generation of a smear layer during tooth-brushing using the dentifrice ${ }^{15)}$. Groups 2, 3, 5, and 6 showed remnants of dentifrice abrasives in the dentinal tubules (Figs. 3C, E, I, and K). Group 4 showed a dentin surface characterized by irregular crystal-like layer deposits leaving many dentinal tubule orifices occluded (Fig. 3G).

\section{Measurement of surface roughness}

The $R a$ value of each group is shown in Table 1. All experimental groups showed an increase in surface roughness in comparison with group 1 . The brushing force produced a rougher surface using the desensitizing dentifrice. Group 1 (control group) showed lower $R a$ values than the other experimental groups, and group 4 (tooth-brushing with a $100-\mu \mathrm{A}$ microcurrent) showed the second-lowest $R a$ value among the groups. Thus, the brushing force does seem to have an effect on the surface roughness and the $100-\mu \mathrm{A}$ microcurrent produced a relatively smoother surface than that observed in the other experimental groups. There was no particular relationship between the ampere and $R a$ values.

\section{Experiment part 2}

1. Measurement of the DFF rate by NFMD

The representative graphs of DFF according to time are shown in Fig. 4. While group 1 shows a constant increase in DFF over time, groups 2 and 4 show a "step- 


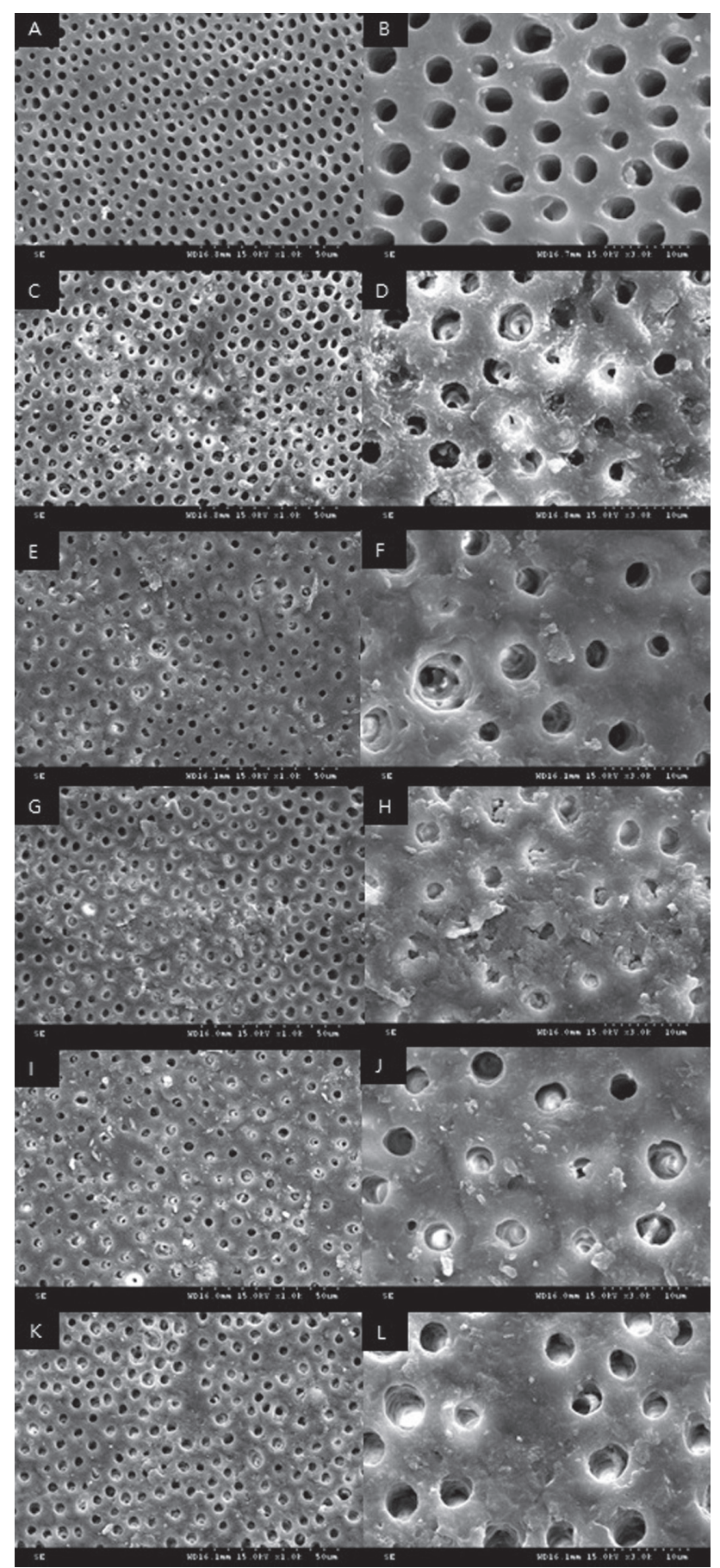

Fig. 3 SEM micrographs of the dentin surface morphology in group 1 at $1,000 \times$ (left) and 3,000× (right) magnifications.

(A and B) Dentin surface after treatment with brushing without a microcurrent $(\mathrm{C}$ and $\mathrm{D})$ and brushing with a $20-\mu \mathrm{A}$ (E and F), 100- $\mu \mathrm{A}(\mathrm{G}$ and $\mathrm{H})$, $200-\mu \mathrm{A}(\mathrm{I}$ and $\mathrm{J})$, and $500-\mu \mathrm{A}$ (K and L) microcurrents at $1,000 \times$ (left) and 3,000× (right) magnifications.

like" increase in DFF while the control group shows a zero value for the DFF rate. The specimens in group 1

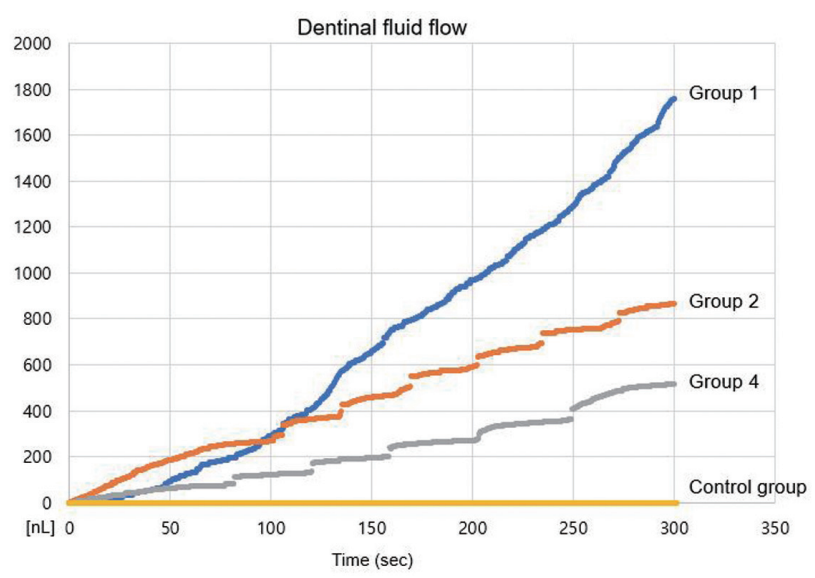

Fig. 4 Representative graphs of dentinal fluid flow in each group.

Group 1: Phosphoric acid-etched specimens without any treatment, group 2: Phosphoric acid-etched specimens brushed without a microcurrent, group 4: Phosphoric acid-etched specimens brushed with a microcurrent $(100 \mu \mathrm{A})$.

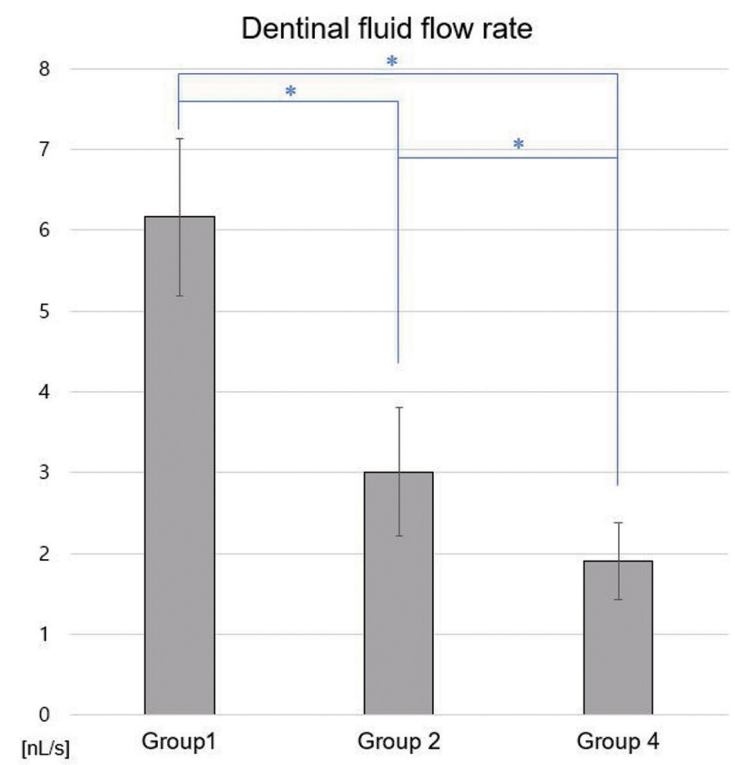

Fig. 5 Bar graph of dentinal fluid flow rates over $5 \mathrm{~min}$. The blue line and asterisk indicate significant differences between groups $(p<0.05)$.

did not receive any treatment except acid etching, so dentinal tubules were open and free of the smear layer, resulting in a constant increase in DFF. In contrast, groups 2 and 4 received tooth-brushing using Colgate Total toothpaste; thus, the dentinal tubules were partially occluded, resulting in a "step-like" increase in the graph. DFF rates of each group and average DFF rates are shown in Fig. 5. Group 4 showed a significantly lower DFF rate than groups 1 and $2(p<0.05)$. 
Table $1 \quad R a$ values of different groups measured by surface profiler

\begin{tabular}{cccccccc}
\hline Group & 1 & 2 & 3 & 4 & 5 & 6 \\
\hline$R a(n m)$ & 319.62 & 427.73 & 445.23 & 358.64 & 627.67 & 506.66 \\
\hline
\end{tabular}

\section{DISCUSSION}

This is the first study to assess the dentinal tubule occluding ability of tooth-brushing with a microcurrent by using an NFMD. While most previous studies on dentin hypersensitivity used a regular toothbrush with desensitizing agents, this study used a smart toothbrush with a microcurrent that had a similar effect as iontophoresis.

Although many hypotheses have been proposed to explain dentin hypersensitivity, the definite principle underlying this phenomenon remains unknown. The most commonly accepted hypothesis for explaining dentin hypersensitivity was proposed in the Brannstrom hydrodynamic theory in 1966 ${ }^{2}$. Therefore, any substance that causes a reduction in DFF by occluding the dentinal tubules can reduce the symptoms of dentin hypersensitivity ${ }^{16)}$. Several in vitro and in vivo studies have already shown the ability of different desensitizing toothpastes to occlude dentinal tubules ${ }^{16,17)}$. Furthermore, iontophoresis has been used to further increase the effectiveness of desensitizing agents, and many studies have shown the effect of iontophoresis in reducing dentin hypersensitivity ${ }^{6,8}$. This study aimed to confirm dentinal tubule occlusion by a smart toothbrush that used a similar principle as iontophoresis.

All experimental groups used a Colgate Total toothpaste, which contains $0.24 \%$ sodium fluoride, when tooth-brushing the dentin specimen. Dentifrice abrasives can have an occluding effect on dentinal tubules. This phenomenon occurs via generation of a smear layer during tooth-brushing using the dentifrice ${ }^{15)}$. Thus, tooth-brushing both with or without microcurrents can occlude dentinal tubules by generating smear layers. In comparison with group 2, groups $3,4,5$, and 6 used microcurrents and were thus expected to show effects similar to those seen with fluoride iontophoresis. Fluoride is known to occlude the dentinal tubule by forming $\mathrm{CaF}_{2}$ crystals $^{18)}$. Some previous studies have reported that the dentinal tubule occlusion effect of fluoride is insignificant and that the crystal structure cannot be observed in SEM images of fluoride-applied dentin specimens ${ }^{19,20)}$. Other clinical studies, on the other hand, reported good effects when using fluoride to treat dentin ${ }^{21,22)}$. Thus, the mechanism and effects of fluoride in dental hypersensitivity appear to be the subject of some controversy. In this study, groups 2, 3, 5 , and 6 did not show a crystal structure but instead showed a resinous plug in the dentinal tubules. This could have occurred because fluoride does not easily form crystals with calcium ions because $\mathrm{CaF}_{2}$ can be easily dissolved as a result of its chemical instability in a humid environment ${ }^{14)}$. In this study, tooth-brushing was performed for $1 \mathrm{~min} /$ day in tap water for 7 days to enhance the effect of dentinal tubule occlusion. After tooth-brushing each day, the dentin specimen was kept in distilled water, and this aspect of the study design possibly created a humid environment that facilitated easy dissolution of $\mathrm{CaF}_{2}$ crystals. Moreover, the toothbrushing force could have affected crystal formation and dentinal tubule occlusion. A previous study by Sehmi et al. compared three brushing forces -100, 200, and $400 \mathrm{~g}$ (low, medium, and high) — and showed significant differences in dentinal tubule patency between the 100and 400-g tooth-brushing forces. At 100-g, formation of a smear layer after tooth brush abrasion occurred, but at $400 \mathrm{~g}$, there were significant increases in dentinal tubule patency, which is clinically relevant for patients with dentin hypersensitivity ${ }^{23)}$. Because of the use of an electric toothbrush in this study, the brushing force may have acted more strongly and interfered with crystal formation. However, group 4 showed a crystal-like layer on the dentin surface in SEM micrographs. This may indicate that the $100-\mu \mathrm{A}$ microcurrent is most effective value to facilitate dentinal tubule occlusion.

There was an increase in the surface roughness of the experimental groups and compared to groups $2,3,5$, 6 , the increase in surface roughness of gorup 4 was the smallest reported any group other than group 1. Thus, the brushing force does seem to have an effect on the surface roughness and the 100- $\mu \mathrm{A}$ microcurrent produced a relatively smoother surface than that observed in the other experimental groups. One possible explanation for this relates to how the $R a$ value is calculated. Surface roughness refers to the height deviation from the form or overall shape of a surface and changes in $R a$ typically signify a new variation in the process ${ }^{24)}$. A limitation of $R a$ is that it provides a quantitative mean of the height deviations and cannot show the surface's irregularities ${ }^{25}$. In group 4 , tooth-brushing with a $100-\mu \mathrm{A}$ microcurrent yielded a crystal-like layer on the dentin surface and occluded the dentinal tubules most effectively. On the basis of this result, the artificial layer created by group 4 may have reduced the height deviations into the exposed patent dentinal tubules and showed a relatively less increase in the $R a$ value. However, there was no particular correlation between the surface roughness and tubule patency across all groups. Although surface roughness was not a direct indicator of tubule patency in this study, surface roughness measurements were useful to understand of the effects of tooth-brushing and the desensitizing dentifrice on the dentin surface.

After SEM and surface roughness analyses, we chose group 4 for DFF rate measurements. To analyze dentinal tubule occlusion quantitatively, the DFF rate was measured using an NFMD. A previous study 
using an NFMD showed that the effect of desensitizing agents could be assessed by estimating the DFF rate ${ }^{14)}$. Many previous studies have investigated the effects of desensitizing agents on dentinal tubule occlusion by assessing dentin permeability on a dentin specimen connected to a capillary in a split chamber ${ }^{9}$. Although this study design has been commonly used because of the convenience of dentin specimen preparation, many studies loaded $70 \mathrm{~cm} \mathrm{H}_{2} \mathrm{O}$ or higher pressures ${ }^{26,27)}$, and these loads were higher than the physiologic pulp pressure to accelerate the movement of the air bubble in the capillary of the flow measurement device ${ }^{14)}$. With an NFMD, a physiologic pulp pressure of $20 \mathrm{~cm} \mathrm{H}_{2} \mathrm{O}$ could be applied, making it possible to reproduce the clinical situation. Moreover, the NFMD has a high resolution, so it can show more details of $\mathrm{DFF}^{13)}$.

There was a significant difference between the mean DFF rate in group 4 compared to those in groups 1 and 2 $(p<0.05)$. Group 4 specimens were brushed using a smart toothbrush, in which a chip consisting of staneless steel and aluminum causes oxidation-reduction reactions with saliva and fluoride-containing toothpaste. Thus, the microcurrent cell and the toothbrushes are electrically connected to supply a microcurrent directly to the teeth with electrolyte, and group 4 can be thought of as having a similar effect as iontophoresis using fluoride. In iontophoresis with fluoride, the fluoride is absorbed under the walls of the dentinal tubules as well as on the surface of calcium, forming calcium fluoride with the tooth substance ${ }^{7}$. This creates a new physical barrier and occludes the dentinal tubules, reducing their permeability. This could probably explain why toothbrushing with a microcurrent yielded the lowest DFF rates among the three groups.

After we investigated dentinal tubule occlusion by measuring the DFF and analyzing SEM micrographs, we concluded that tooth-brushing with microcurrents was effective in occluding the dentinal tubules. However, it may be difficult for the desensitizing agent to be retained on an exposed dentin surface because of interactions with saliva and cyclic brushing in clinical situation. And unlike clinical situation, dentin surface was tooth-brushed in the tap water during the experiment. Therefore, extrapolation of these results to the clinical situation should be performed carefully. Moreover, there is limited clinical assessment data for dentin hypersensitivity. Further clinical and long-term studies using microcurrents should be performed to determine the effect of tooth-brushing with microcurrents on reducing dentin hypersensitivity.

\section{CONCLUSIONS}

Within the limitations of this study, the following conclusions were reached:

1) All experimental groups showed partially occluded dentinal tubules and the formation of crystal-like structures at a specific microcurrent intensity indicated that tooth-brushing with microcurrents could occlude dentinal tubules efficiently.
2) The decrease in DFF rate in the tooth-brushing with microcurrent groups indicated that dentinal tubules were occluded and that the flow of dentinal fluid had decreased.

\section{ACKNOWLEDGMENTS}

This study was performed using equipment donated by Microworld. However, the authors of this paper certify that they have no proprietary, financial, or other personal interest in this study. This work was supported by the National Research Foundation of Korea grant funded by the Korea government (NRF-2018R1A1A1A05018328).

\section{REFERENCES}

1) Orchardson R, Gillam DG. Managing dentin hypersensitivity. J Am Dent Assoc 2006; 137: 990-998; quiz 1028-1029.

2) Brannstrom M, Linden LA, Johnson G. Movement of dentinal and pulpal fluid caused by clinical procedures. J Dent Res 1968; 47: 679-682.

3) Wang Z, Sa Y, Sauro S, Chen H, Xing W, Ma X, et al. Effect of desensitising toothpastes on dentinal tubule occlusion: a dentine permeability measurement and SEM in vitro study. J Dent 2010; 38: 400-410.

4) Ling TY, Gillam DG, Barber PM, Mordan NJ, Critchell J. An investigation of potential desensitizing agents in the dentine disc model: a scanning electron microscopy study. J Oral Rehabil 1997; 24: 191-203.

5) Herndon CM. Iontophoretic drug delivery system: focus on fentanyl. Pharmacotherapy 2007; 27: 745-754.

6) Patil AR, Varma S, Suragimath G, Abbayya K, Zope SA, Kale V. Comparative evaluation of efficacy of iontophoresis with $0.33 \%$ sodium fluoride gel and diode laser alone on occlusion of dentinal tubules. J Clin Diagn Res 2017; 11: ZC123-ZC126.

7) Wilson JM, Fry BW, Walton RE, Gangarosa LP Sr. Fluoride levels in dentin after iontophoresis of $2 \% \mathrm{NaF}$. J Dent Res 1984; 63: 897-900.

8) Carlo GT, Ciancio SG, Seyrek SK. An evaluation of iontophoretic application of fluoride for tooth desensitization. J Am Dent Assoc 1982; 105: 452-454.

9) Vieira AH, Santiago SL. Management of dentinal hypersensitivity. Gen Dent 2009; 57: 120-126; quiz 127-128.

10) Austin RS, Mullen F, Bartlett DW. Surface texture measurement for dental wear applications. Surf Topogr 2015; 3: 023002

11) Hara AT, Livengood SV, Lippert F, Eckert GJ, Ungar PS. Dental surface texture characterization based on erosive tooth wear processes. J Dent Res 2016; 95: 537-542.

12) Olley RC, Moazzez R, Bartlett D. Effects of dentifrices on subsurface dentin tubule occlusion: an in situ study. Int $\mathrm{J}$ Prosthodont 2015; 28: 181-187.

13) Kim SY, Ferracane J, Kim HY, Lee IB. Real-time measurement of dentinal fluid flow during amalgam and composite restoration. J Dent 2010; 38: 343-351.

14) Kim SY, Kim EJ, Kim DS, Lee IB. The evaluation of dentinal tubule occlusion by desensitizing agents: a realtime measurement of dentinal fluid flow rate and scanning electron microscopy. Oper Dent 2013; 38: 419-428.

15) Prati C, Venturi L, Valdre G, Mongiorgi R. Dentin morphology and permeability after brushing with different toothpastes in the presence and absence of smear layer. J Periodontol 2002; 73: $183-190$.

16) Markowitz K, Pashley DH. Discovering new treatments for sensitive teeth: the long path from biology to therapy. J Oral Rehabil 2008; 35: 300-315.

17) Arrais CAG, Micheloni CD, Giannini M, Chan DCN. Occluding 
effect of dentifrices on dentinal tubules. J Dent 2003; 31: 577 584.

18) Greenhill JD, Pashley DH. The effects of desensitizing agents on the hydraulic conductance of human dentin in vitro. J Dent Res 1981; 60: 686-698.

19) Pashley DH. Dentin permeability, dentin sensitivity, and treatment through tubule occlusion. J Endod 1986; 12: 465474.

20) Paes Leme AF, dos Santos JC, Giannini M, Wada RS. Occlusion of dentin tubules by desensitizing agents. Am J Dent 2004; 17: 368-372.

21) Thrash WJ, Jones DL, Dodds WJ. Effect of a fluoride solution on dentinal hypersensitivity. Am J Dent 1992; 5: 299-302.

22) Yates RJ, Newcombe RG, Addy M. Dentine hypersensitivity: a randomised, double-blind placebo-controlled study of the efficacy of a fluoride-sensitive teeth mouthrinse. J Clin
Periodontol 2004; 31: 885-889.

23) Sehmi H, Olley RC. The effect of toothbrush abrasion force on dentine hypersensitivity in-vitro. J Dent 2015; 43: 14421447.

24) Gadelmawla ES, Koura MM, Maksoud TMA, Elewa IM, Soliman HH. Roughness parameters. J Mater Process Technol 2002; 123: 133-145.

25) Field J, Waterhouse P, German M. Quantifying and qualifying surface changes on dental hard tissues in vitro. J Dent 2010; 38: $182-190$.

26) Elgalaid TO, Creanor SL, Creanor S, Hall AF. The permeability of natural dentine caries before and after restoration: An in vitro study. J Dent 2007; 35: 656-663.

27) Pashley DH, Matthews WG, Zhang Y, Johnson M. Fluid shifts across human dentine in vitro in response to hydrodynamic stimuli. Arch Oral Biol 1996; 41: 1065-1072. 